\title{
Queueing Game For Spectrum Access in Cognitive Radio Networks
}

\author{
Zheng Chang, Member, IEEE, Tapani Ristaniemi, Senior Member, IEEE, Zhu Han, Fellow, IEEE,
}

\begin{abstract}
In this paper, we investigate the problem of spectrum access decision-making for the Secondary Users (SUs) in the cognitive radio networks. When the Primary Users (PUs) are absent on certain frequency bandwidth, SUs can formulate a queue and wait for the Base Station (BS) to serve. The queue of the SUs will be dismissed if the PU is emerging in the system. Leveraging the queueing game approaches, the decision-making process of the SUs that whether to queue or not is studied. Both individual equilibrium and social optimization strategies are derived analytically. Moreover, the optimal pricing strategy of the service provider is investigated as well. Our proposed algorithms and corresponding analysis are validated through simulation studies.
\end{abstract}

Index Terms-queueing game; pricing; Nash equilibrium strategies; social optimizations; spectrum access; cognitive radio

\section{INTRODUCTION}

In the fast growing wireless market, the frequency spectrum is one of the most scarce and valuable resources. However, some surveys for actual measurements show that most of the allocated spectrum is largely under-utilized [1]. Cognitive radio $(\mathrm{CR})$ is known as an efficient way to improve spectrum utilization and a promising technology to enable dynamic spectrum access by exploiting the unused spectrum in the wireless environments [1]. In a cognitive radio network (CRN), there are two types of users, namely, licensed primary users (PUs) who have the licensed spectrum access opportunities and unlicensed secondary users (SUs) who can only utilize the spectrum which the PUs do not occupy. To exploit limited spectrum efficiently, In a CRN, the SUs are allowed to opportunistically to access licensed spectrum bands when PU transmission is not presented, which is able to significantly improve the spectrum utilization efficiency.

During last decades, the technology of CR has attracted plenty of interests and has been investigated extensively, among which, how to explore the access opportunities and regular the spectrum access without harming the PUs are of consistent research interests [2]. Recently, there is a particular interest to apply the queueing theory [3] which is a natural tool to analyze the transmission in the wireless networks or the game theory [5] which is commonly used in developing optimization algorithms to address the spectrum access problems. Intriguingly, applying queue theory with pricing strategies also brings a novel view on understanding the SU's behavior

Z. Chang and T. Ristaniemi are with University of Jyväkylä, Department of Mathematical Information Technology, P.O.Box 35, FIN-40014 Jyväkylä, Finland. Z. Han is with the Electrical and Computer Engineering Department, University of Houston, Houston, USA. E-mail: zheng.chang@jyu.fi, zhan2@uh.edu, tapani.ristaniemi@jyu.fi and cognitive market policies, which can be traced from the original works of [6]-[9] that study the equilibrium behavior in queueing systems. We call it the queueing game in the following to highlight the key issues in this area: by utilizing the information of queue and considering its own payoff, the user needs to make the decision on whether to queue or not. Recent works of [10]-[12] have leveraged the queueing game to the spectrum access control in the CRN. In [10], the authors present an observed queue model with SUs acquiring for transmission from a cognitive base station (CBS). It is also assumed that PUs emerged periodically for transmission opportunities. During the PU's transmission period, CBS stops serving SUs and SUs remained in the queue waiting for the re-operation of CBS. Based on this model, a SU's decision strategy, i.e., joining the queue or balk, is investigated and the pricing policy was studied. Authors of [11] extend the work of [10] to an unobserved case and analyzed the strategies of SUs and CBS. In [12], the strategy of delay sensitive SUs is explicitly considered. The authors also consider pricing and load balancing effect in the spectrum access decision-making in both monopoly and duopoly markets. Authors of [13] also utilizes the concept of the queueing game and model users as selfish players that compete with each other by choosing the optimal transmission threshold maximizing system throughput.

It can be well observed that the queueing game is an effective tool for analyzing the SUs' behaviors in spectrum access. The motivation of this work is to extend the previous works and overcome their limitations. It can be found that the previous works focused on the case that a separate CBS is used for serving the SUs. Therefore, when the PU emerges, the SUs can remain in the queue of the CBS and wait for PUs finishing the transmission. In such case, the queue of the CBS can be modeled and analyzed by a server breakdown model. However, the installation and deployment of the CBS bring additional cost and it may not be practical in some cognitive systems. In contrast, we consider there is only one BS in the system. The primary job of the BS is to serve the incoming PUs, while it will utilize the time when the PU frequency band is not occupied to serve SUs. Specially, when the PU comes, the BS no long holds the information of the queue and all the SUs are forced to leave the queue. When a SU decides to join the queue and wait for spectrum access opportunity, the sojourn time induces a cost and if its job has been finished, the SU can receive a reward. In addition, if PU emerges, the SUs who have to leave the queue without any reward or compensate. Under this model, we study the SU's decision-making process, i.e., whether to join for spectrum access or balk, and the pricing based spectrum access control. 
Compared to previous works, the main contributions of this paper are as follows. We first model the interaction among SUs in a partially observed queue as a noncooperative game. Based on the queueing analysis and payoff model, we then analyze both individually equilibrium strategy and optimal social welfare strategy of the SUs about whether to join or not. Furthermore, we study the BS pricing strategy for the system such that the individually equilibrium decision of SUs can coincide with the socially optimal strategy that optimizes the total welfare of the whole system. Our presented analysis and algorithm are demonstrated by the simulation studies.

The rest of this paper is organized as follows. Section II describes the system model including the queue model and profit of SUs. We present the individual equilibrium strategies, social welfare optimization and pricing studies in Section III. Our presented algorithm and analysis are demonstrated in Section IV through simulation studies, and finally we conclude this work in Section V.

\section{SYSTEM MODEL}

\section{A. Queue Model}

The system model can be found in Fig. 11 We consider a CRN consists of multiple SUs, one BS and multiple PUs. When PUs are absent, the BS can utilize the available spectrum to serve the SUs. If the PU is entering the system, due to its priority, the BS has to drop the service connection of the SUs and starts to serve PU. Meanwhile, the queue consisting of the SUs is no long exists and all SUs should leave the queue and seek for other transmission opportunities. We consider the data arrival rate of the SU follows the Poisson process at rate $\lambda$ and the arrival of the PU follows Poisson process at rate $\xi$. The service requirements of the SUs are i.i.d with exponential distribution $\mu$. The First-Come-First-Served (FCFS) rule is applied for determining the service order of SUs at the BS. The length of the service time of the PU is also assumed to be exponentially distributed with rate $\eta$. The above considered queue model is a common assumption in some previous literatures, e.g. [10] [11].

The state of the system at time $t$ is represented by a pair $(N(t), I(t))$, where $N(t)$ is the length of queue, i.e., the number of SUs in the system. $I(t)$ denotes the working status of the BS, with 1 standing for serving SUs and 0 showing that the BS is serving the PUs. So based on the system model, when $I(t)=0$, we also have $N(t)=0$. We assume when being successfully served by the BS, the SU can receive a reward. While waiting in the queue, the delay cost of the $\mathrm{SU}$ is a function of its sojourn time. Based on the total payoff which is the difference between the reward and the cost, SU can make the decision on whether to join the queue and waiting for spectrum access or not. The payoff models are presented in the following.

\section{B. Profit Model}

We assume when being successfully served by the BS, the SU receives a reward $\varpi_{s}$. We also assume that the cost of staying in the queue is $\chi(T)$ where $T$ is the sojourn time in the system representing the time that SU stays in the queue.

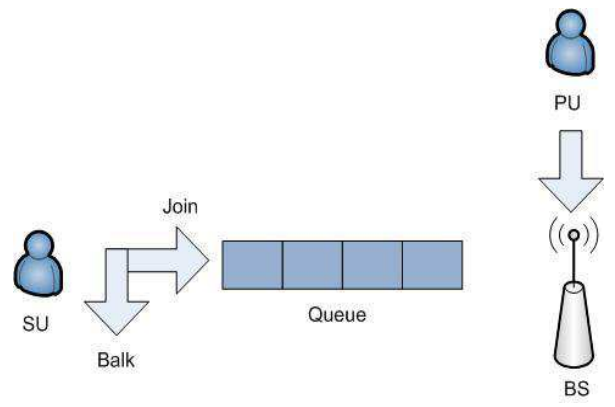

Fig. 1. System Model

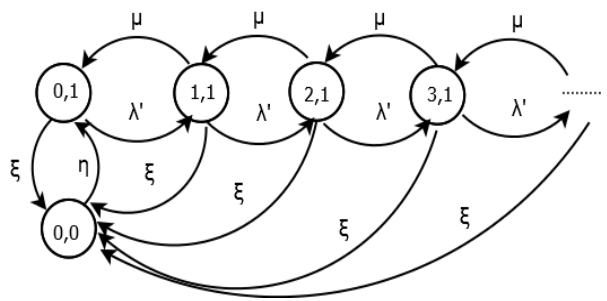

Fig. 2. Transition-rate diagram

$\chi(T)$ should be an increasing function of $T$ and one simple linear example is that $\chi(T)=C T$ where $C$ is the unit cost. Then the profit of the SU can be given as

$$
R:=\varpi_{s}-C T .
$$

Accordingly, we can have the definition of the expected individual profit

Definition 1. When there are $n$ SUs in the queue, the expected profit of the arriving $\mathrm{SU}$ can be defined as

$$
U(n):=\theta_{s}\left(\varpi_{s}-C E\left(Q_{n}\right)\right),
$$

where $\theta_{s}$ is the probability that the SU can be served and $E\left(Q_{n}\right)$ is the expected sojourn time related to $n$.

Moreover, we can also define the social welfare as follows.

Definition 2. The social welfare of the considered system is given by

$$
S(q):=\lambda \rho_{s} \varpi_{s}-C E(N),
$$

where $\rho_{s}$ is the fraction of SUs that join the queue and leave after being served. $E(N)$ is the mean number of SUs.

In the following, we consider the SUs are risk natural and try to maximize their profits. We also assume the SUs are identical i.e., a symmetric game is assumed. In addition, we also consider that when the SU enters the system at time $t$, all the system parameters including profit model are known except the queue length $N(t)$ and SU can only observe $I(t)$ upon arrival, i.e., a partially observed queue is considered.

\section{Queueing Game for SPectrum Access: EQUILIBRIUM AND PRICING}

\section{A. Stationary Probability and Expected Sojourn Time}

After entering the system, the SU is able to decide whether to join the queue waiting for spectrum access or not based 
on its own observation, i.e. the profit. Thus, there are only two pure strategies, join and balk, and a mixed strategy is specified by the joining probability $q \in[0,1]$ of a SU that finds the BS is not serving PU. Therefore, the effective arrival rate is $\lambda^{\prime}=\lambda q$. For the considered system, we first obtain the stationary probabilities $p(N(t), I(t))$. Based on the transition rate diagram in Fig. 2, we are able to obtain the stationary probabilities $p(N(t), I(t))$ based on the following balance equations,

$$
\begin{gathered}
\eta p(0,0)=\xi \sum_{n=0}^{+\infty} p(n, 1) \\
\left(\lambda^{\prime}+\xi\right) p(0,1)=\mu p(1,1)+\eta p(0,0) \\
\left(\lambda^{\prime}+\xi+\mu\right) p(n, 1)=\lambda^{\prime} p(n-1,1)+\mu p(n+1,1), \forall n \geq 1, \\
p(0,0)+\sum_{n=0}^{+\infty} p(n, 1)=1 .
\end{gathered}
$$
tion.

To obtain $p(0,0)$ and $p(n, 1)$, we have following observa-

Proposition 1. The stationary probability $p(0,0)$ and $p(n, 1)$ can be given as follows,

$$
\begin{aligned}
p(0,0) & =\frac{\xi}{\eta+\xi}, \\
p(n, 1) & =\frac{\eta\left(1-x\left(\lambda^{\prime}\right)\right) x\left(\lambda^{\prime}\right)^{n}}{\eta+\xi}
\end{aligned}
$$

where $x\left(\lambda^{\prime}\right)$ is

$$
x\left(\lambda^{\prime}\right)=\frac{\left(\lambda^{\prime}+\mu+\xi\right)-\sqrt{\left(\lambda^{\prime}+\mu+\xi\right)^{2}-4 \lambda^{\prime} \mu}}{2 \mu} .
$$

Proof: The proof is given in Appendix A.

In order to achieve the expected profit, from (2), $\theta_{s}$ and $E\left(Q_{n}\right)$ should be obtained at the first place. Consider a SU enters the network at state $(n, 1)$ upon arrival and decides to join the queue. This SU may leave either due to service completion or due to a PU entering. For its service completion, the SU has to wait for a sum of $n+1$ independent exponentially distributed times with parameter $\mu$. For the case that the PU enters, the SU has to wait for an exponentially distributed time with parameter $\xi$. Hence, the sojourn time of the SU in the system is given as $N=\min \left(L_{n}, Q\right)$, where $L_{n}$ follows a Gamma distribution with parameters $n+1$ and $\mu$. $Q$ is an exponentially distributed random variable with rate $\xi$, and $Q$ is independent of $L_{n}$. Therefore, we have $\theta_{s}=\operatorname{Pr}\left(L_{n}<Q\right)$. To this end, when considering $N(t)$ and $I(t)$ are known to the SU, we can obtain the $\theta_{s}$ and $E\left(Q_{n}\right)$ in (2) as

$$
\begin{aligned}
\theta_{s} & =\left(\frac{\mu}{\mu+\xi}\right)^{n+1}, \\
E\left(Q_{n}\right) & =\frac{1}{\xi}\left(1-\theta_{s}\right)^{n+1},
\end{aligned}
$$

where $n$ is the number of SUs in the queue. Then, we can use (4) and (10) to address $U(n)$ in (2) and then find the expected profit of a SU that enters the queue with a certain probability.

\section{B. Individually Equilibrium Strategy}

With the results of the stationary probability and the expected sojourn time, the expected profit of a SU that enters the queue with probability $\tilde{q}$ can be achieved as following,

Proposition 2. When there is frequency bandwidth available for the SUs, the expected profit of a SU that enters the queue with probability $\tilde{q}$ given that other SUs join the queue with probability $q$ is given by

$$
\Gamma(\tilde{q}, q)=\tilde{q}\left[\left(\varpi_{s}+\frac{C}{\xi}\right) \frac{\mu\left(1-x\left(\lambda^{\prime}\right)\right)}{\mu+\xi-\mu x\left(\lambda^{\prime}\right)}-\frac{C}{\xi}\right]
$$

Proof: The proof is given in Appendix B.

We can now process to find out the individually equilibrium (IE) strategy of a SU and we have the following,

Theorem 1. In the considered model, a unique IE mixed strategy exists, with joining probability $q_{e}$ given by

$$
q_{e}= \begin{cases}1, & \varpi_{s} \in\left[\frac{C}{\mu(1-\kappa)},+\infty\right), \\ \Theta, & \varpi_{s} \in\left(\frac{C}{\mu}, \frac{C}{\mu(1-\kappa)}\right), \\ 0, & \text { Otherwise. }\end{cases}
$$

where $\Theta=\frac{\left(\mu \varpi_{s}-C\right)\left(C+\xi \varpi_{s}\right)}{\lambda C \varpi_{s}}$ and $\kappa=x(\lambda)$.

Proof: The proof is given in Appendix C.

From Theorem 1, we can observe that the IE is independent of the PU's service time. This is because the SU makes decision only when PU is not being served and the SU can not be aware of the PU's information. However, the socially equilibrium strategies of the SUs do depend on the pricing strategies of the BS as well as the PU's arrival rate. The IE does not imply the social welfare optimality. Thus, in the following, we investigate the social optimal strategy.

\section{Social Optimization}

Based on the definition of social welfare and previous results of the stationary probabilities, we can arrive at the following proposition,

Proposition 3. The expected social profit, given that the SUs follow a mixed strategy with probability $q$ of joining (i.e. arriving SUs that find an BS not serving the PU enters with probability $q$, while the rest choose to balk without being served) is given by

$$
S(q)=\frac{\eta x\left(\lambda^{\prime}\right)\left[\mu \varpi_{s}\left(1-x\left(\lambda^{\prime}\right)\right)-C\right]}{(\xi+\eta)\left(1-x\left(\lambda^{\prime}\right)\right)} .
$$

Proof: To address the social welfare (3), we need to find $E(N)$ and $\rho_{s}$. Using (4) and (10), we can obtain $\rho_{s}$ and $E(N)$ as 


$$
\begin{aligned}
& \rho_{s}=\sum_{n} p(n, 1) q\left(\frac{\mu}{\mu+\xi}\right)^{(n+1)}, \\
& E(N)=\sum_{n} n p(n, 1) .
\end{aligned}
$$

Then, obtaining the geometric sums in (14) and through (3) we can arrive (13).

With the observation in (13), we can obtain the socially optimal strategy, which can be found in the following theorem.

Theorem 2. In the considered model, a unique socially optimal strategy exists with probability $q_{s}$ of joining the queue which can be expressed as

$$
q_{s}= \begin{cases}1, & \varpi_{s} \in\left[\frac{C}{\mu(1-\kappa)^{2}},+\infty\right), \\ \Phi, & \varpi_{s} \in\left(\frac{C}{\mu}, \frac{C}{\mu(1-\kappa)^{2}}\right), \\ 0, & \text { Otherwise. }\end{cases}
$$

where $\Phi=\frac{\sqrt{\vartheta}\left(\mu \varpi_{s}-\sqrt{\vartheta}\right)\left(\xi \varpi_{s}+\sqrt{\vartheta}\right)}{\lambda \vartheta \varpi_{s}}$ and $\vartheta=\mu \varpi_{s} C$.

Proof: The proof is given in Appendix D.

\section{Optimal Pricing}

We have obtained the socially optimal strategy as well as the individual equilibrium strategy. Moreover, it can be observed that the socially optimal joining probability $q_{s}$ is always smaller than the individual one $q_{e}$, which can also be found in Fig. 3. To oblige the SUs to adopt the socially optimal strategy, one approach is to apply a pricing mechanism to reduce the individually optimal threshold $q_{e}$ [10]. In this work, we consider that the BS will act as an agent to impose an admission fee, which is a constant given the arrival rate, service pattern, reward and cost. The admission fee is to force the individually equilibrium probability to equal with the social optimal one.

When the admission fee is considered, the expected profit of a SU is given as $U(n, p)=\theta_{s}\left(\varpi_{s}-C E\left(Q_{n}\right)-p\right)$. It can be observed that when imposing an admission fee, the social profit remains the same as it implies a transfer of income from one group to another. Thus, through 21, we can obtain $\Gamma\left(q_{s}, q_{s}, p\right)$. We further denote that the equilibrium probability of joining by $q_{e}(p)$. Then the optimal fee $p^{*}$ should satisfy $q_{s}=q_{e}\left(p^{*}\right)$. As the monopoly market is considered here, and a monpoly does not allow the a positive user surplus since in such a case, the price can be increased without reducing $q$. Therefore, the $p^{*}$ can be arrived by

$$
p^{*}=\left\{p \mid \Gamma_{p}\left(q_{s}, q_{s}, p\right)=0\right\},
$$

\section{Simulation Results}

The numerical parameters are $\lambda=7, \xi=0.5, \mu=3, \eta=$ $2, C=2$. First, in Fig. 3 we can see that the socially optimal joining probability $q_{s}$ is always smaller than the individual one $q_{e}$, and inherently, there is a gap between the individually equilibrium arrival rate and social arrival rate as the equilibrium arrival rate and social arrival rate can be given as $\lambda_{e / s}=\lambda q_{e / s}$. Therefore, we can see that by imposing

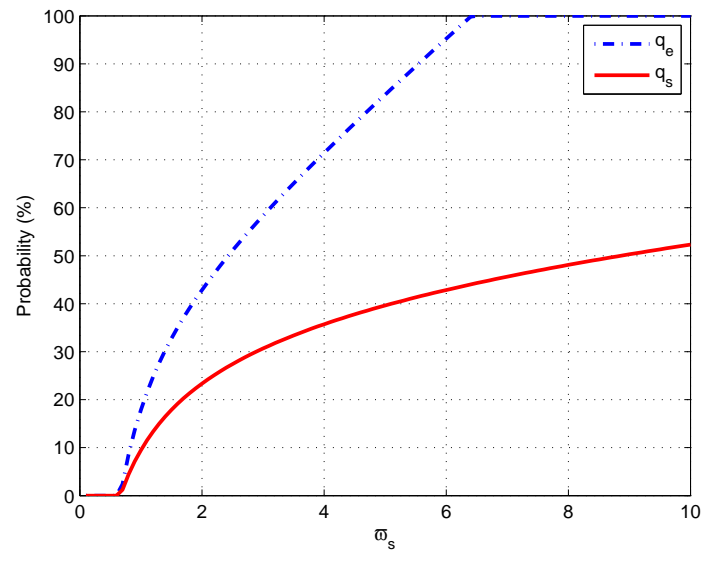

Fig. 3. The equilibrium joining probability vs. reward

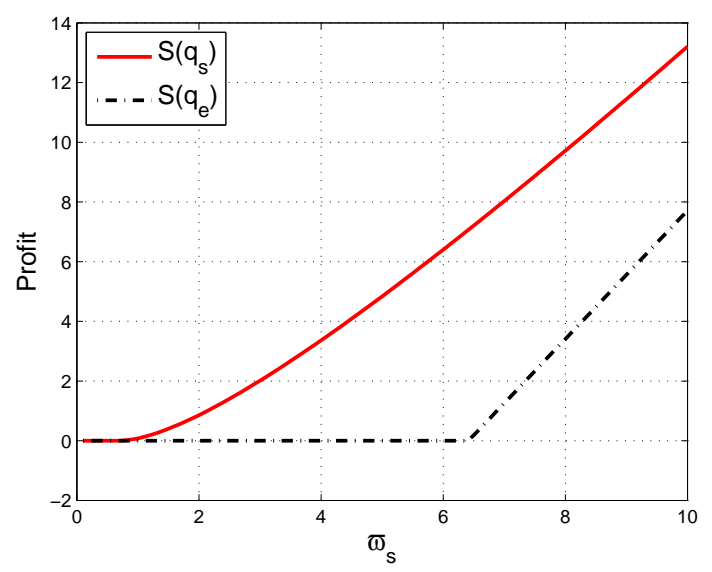

Fig. 4. The profits vs. reward

appropriate admission fee, the arrival rate of SUs can be regulated. In Fig. 4, we can see that the social benefit when $q_{s}$ is used is higher than the one when $q_{e}$ is considered. Comparing Fig. 3 and Fig. 4 we can see that only when $q_{e}=1$, the social benefit is higher than 0 , otherwise it remains lower or equal to 0 . Meanwhile, the social benefits when $q_{s}$ is considered is always above 0 and comparable high, which indicates that appropriate admission fee can improve the social benefits of the considered system.

\section{CONCLUSION}

In this paper, we have investigated the problem of spectrum access decision-making for the SUs in the CRNs. Utilizing the queueing game approaches, the decision-making process of the SUs that whether to queue or not is studied in present of arrival of the PUs. Both individual equilibrium and social optimization strategies are derived. Moreover, the optimal pricing of the service provider is also investigated. Our proposed algorithms and corresponding analysis are validated through simulation studies.

\section{APPENDIX A}

From (4)-(7), we can observe $p(0,0)=\frac{\xi}{\eta+\xi}$. To obtain $p(n, 1)$, the similar approach used in [8, pp. 578] can be 
applied. One can notice that (6) can be considered as a homogeneous linear difference equation of order 2 , which is with constant coefficients and characteristic equation

$$
\left(\lambda^{\prime}+\mu+\xi\right) y=\lambda^{\prime}+\mu y^{2} .
$$

(17) have too roots, $x^{\prime}\left(\lambda^{\prime}\right)$ and $x\left(\lambda^{\prime}\right)$, which are

$$
\begin{gathered}
x^{\prime}\left(\lambda^{\prime}\right)=\frac{\left(\lambda^{\prime}+\mu+\xi\right)+\sqrt{\left(\lambda^{\prime}+\mu+\xi\right)^{2}-4 \lambda^{\prime} \mu}}{2 \mu}, \\
x\left(\lambda^{\prime}\right)=\frac{\left(\lambda^{\prime}+\mu+\xi\right)-\sqrt{\left(\lambda^{\prime}+\mu+\xi\right)^{2}-4 \lambda^{\prime} \mu}}{2 \mu} .
\end{gathered}
$$

From the standard theory of homogeneous linear difference equations (see e.g. [14, Sec 2.3]), we conclude that

$$
p(n, 1)=c^{\prime} x^{\prime}\left(\lambda^{\prime}\right)^{n}+c x\left(\lambda^{\prime}\right)^{n},
$$

where $c^{\prime}$ and $c$ are constants. We can easily see that $x^{\prime}>$ 1 , thus, $c^{\prime}$ should be necessarily 0 . The constant $c$ can be calculated using (7) and Proposition 1 can be approved.

\section{APPENDIX B}

To prove Proposition 2, first we have the expected profit when a SU is able to observe $n$ SUs and queue state in the system upon arrival (i.e. observed queue) and decide to enter, which can be derived from (10),

$$
U(n)=\varpi_{s}\left(\frac{\mu}{\mu+\xi}\right)^{(n+1)}-\frac{C}{\xi}+\frac{C}{\xi}\left(\frac{\mu}{\mu+\xi}\right)^{(n+1)}
$$

When a SU decides to join given that the BS is serving SUs and others are following strategy $q$, the expected profit is

$$
\begin{aligned}
\Gamma(1, q) & =\sum_{n=0}^{+\infty} p_{1}(n, 1) U(n) \\
& =\sum_{n=0}^{+\infty} \frac{p(n, 1)}{\sum_{i=0}^{+\infty} p(i, 1)} U(n) \\
& =\left(\varpi_{s}+\frac{C}{\xi}\right) \frac{\mu\left(1-x\left(\lambda^{\prime}\right)\right)}{\mu+\xi-\mu x\left(\lambda^{\prime}\right)}-\frac{C}{\xi},
\end{aligned}
$$

where $p_{1}(n, 1)=\frac{p(n, 1)}{\sum_{i=0}^{+\infty} p(i, 1)}$ is the probability that there are $n$ SUs in the queue when a SU arrives. As we have that $\Gamma(\tilde{q}, q)=$ $(1-\tilde{q}) \Gamma(0, q)+\tilde{q} \Gamma(1, q), 11$ can be arrived and Proposition 2 can be proved.

\section{APPENDIX C}

For a SU, it will prefer to enter the queue if its expected profit after entering $\Gamma(1, q)>0$, and balk if it $\Gamma(1, q)<$ 0 . Considering $\Gamma(1, q)=0$, we can solve for $x\left(\lambda^{\prime}\right)$ and the unique solution is $x=1-\frac{C}{\mu \varpi_{s}}$.

We notice that $x\left(\lambda^{\prime}\right), \lambda^{\prime}=\lambda q$ is one root of (17). Then we are able to solve (17) with respect to $q$, which yields

$$
q_{e}=\frac{x[\mu(1-x)+\xi]}{\lambda(1-x)}=\frac{\left(\mu \varpi_{s}-C\right)\left(C+\xi \varpi_{s}\right)}{\lambda C \varpi_{s}}
$$

We can also observe that $x\left(\lambda^{\prime}\right)$ is a strictly increasing function for $q \in[0,1]$ as $\frac{d x\left(\lambda^{\prime}\right)}{d q}>0$. Thus, $q_{e} \in(0,1)$ iff $x\left(\lambda q_{e}\right) \in(0, \kappa)$, where $\kappa=x(\lambda)$. In other word, $q_{e}$ is in the interval $(0,1)$ iff $\varpi_{s} \in\left(\frac{C}{\mu}, \frac{C}{\mu(1-\kappa)}\right)$.

Moreover, it can be found that when $\varpi_{s} \geq \frac{C}{\mu(1-\kappa)}, \Gamma(1, q)$ keeps positive. Thus, the SU's best response is to join. In this case, "join" is the unique individual equilibrium. On the other hand, when $\varpi_{s} \leq \frac{C}{\mu}, \Gamma(1, q)$ becomes negative. In this case, "balk" is the individuals equilibrium strategy. Therefore, Theorem 1 can be proved.

\section{APPENDIX D}

It can be noticed that (13) can be reformed as $S(q)=$ $f(x(\lambda q))$, where $f(x)=\frac{\eta x\left[m u \varpi_{s}(1-x)-C\right]}{(\xi+\eta)(1-x)}$. As we can see, $S^{\prime}(q)=0$ can be deduced to $f^{\prime}(x(\lambda q))=0$, which means that we need to solve $f^{\prime}(x)=0$, that is

$$
\mu \varpi_{s} x^{2}-2 \mu \varpi_{s} x+\mu \varpi_{s}-C=0 .
$$

We can see that the discriminant of the quadratic polynomial in (23) is less or equal to 0 iff $C \leq 0$. We can deduce that $f(x)$ is increasing and so as $S(q)$. (23) has two roots, which are

$$
x_{1}=1-\frac{\sqrt{\vartheta}}{\mu \varpi_{s}}, x_{2}=1+\frac{\sqrt{\vartheta}}{\mu \varpi_{s}} .
$$

It is apparently $x_{1}<1<x_{2}$ and we have following discussions,

- When $x_{1} \leq 0$, then we have $x_{1}<0<\kappa<x_{2}$. Then $\mathrm{S}(\mathrm{q})$ is decreasing in $[0,1]$ and the social optimal is $q_{s}=0$. We can also see that $x_{1} \leq 0$ implies that $\varpi_{s} \leq \frac{C}{\mu}$.

- When $0<x_{1}<\kappa$, then we can see that the maximum $S(q)$ is obtained for $q$ such that $x(q)=x_{1}$. By using (17), substituting $x_{1}$ for $y$ and solving for $q$, we obtain $q_{s}=\frac{\sqrt{\vartheta}\left(\mu \varpi_{s}-\sqrt{\vartheta}\right)\left(\xi \varpi_{s}+\sqrt{\vartheta}\right)}{\lambda \vartheta \varpi_{s}}$. It can be observed that $0<$ $x_{1}<\kappa$ means $\frac{C}{\mu}<\varpi_{s}<\frac{C}{\mu(1-\kappa)^{2}}$.

- Similarly, when $x_{1} \geq \kappa$ we can have the social optimal joining probablity is $q_{s}=1$ and $\varpi_{s} \geq \frac{C}{\mu(1-\kappa)^{2}}$.

\section{REFERENCES}

[1] E. Hossain, D. Niyato, and Z. Han, Dynamic Spectrum Access and Management in Cognitive Radio Networks. Cambridge University Press Cambridge, 2009.

[2] Q. Zhao, and B. M. Sadler, "A survey of dynamic spectrum access," IEEE Signal Processing Magazine, vol. 24, no. 3, pp. 79-89, May 2007.

[3] H.-P. Shiang and M. van der Schaar, "Queuing-based dynamic channel selection for heterogeneous multimedia applications over cognitive radio networks," IEEE Trans. Multimedia, vol. 10, no. 5, pp. 896-909, Aug. 2008.

[4] L. Yang, H. Kim, J. Zhang, M. Chiang, and C. W. Tan, "Pricingbased decentralized spectrum access control in cognitive radio networks," IEEE/ACM Trans. Networking, vol. 21, no. 2, pp. 522-535, Apr. 2013.

[5] Z. Han, D. Niyato, W. Saad, T. Basar and A. Hjoungnes, Game Theory in Wireless and Communication Networks: Theory, Models, and Applications. Cambridge University Press, 2011.

[6] P. Naor, "The regulation of queue size by levying tolls," Econometrica, vol. 37, no. 1, pp. 15-24,Jan. 1969.

[7] R. Hassin, and M. Haviv, To queue or not to queue: Equilibrium Behavior in Queueing Systems, Springer, New York, 2003.

[8] O. Boudali, and A. Economou, "The effect of catastrophes on the strategic customer behavior in queueing systems," Naval Research Logistics, vol 60, no. 7, pp. 571-587, Oct. 2013. 
[9] E. Altman, "Applications of dynamic games in queues," in Advances in Dynamic Games. Boston, MA: Birkhauser, 2005.

[10] H. Li and Z. Han, "Socially optimal queuing control in CR networks subject to service interruptions: to queue or not to queue?" IEEE Trans. Wireless Commun., vol. 10, no. 5, pp. 1656-1666, May 2011.

[11] C. T. Do, N. H. Tran, M. V. Nguyen, C. S. Hong, and S. Lee, "Social optimization strategy in unobserved queueing systems in cognitive radio networks," IEEE Commun. Lett., vol. 16, no. 12, pp. 1944-1947, Dec. 2012.

[12] N. H. Tran, C. S. Hong, S. Lee, and Z. Han, "Optimal pricing effect on equilibrium behaviors of delay-sensitive users in cognitive radio networks," IEEE J. Sel. Areas Commun., vol. 31, no. 11, pp. 2566-2579, Nov. 2013.

[13] Z. Guan, T. Melodia, and G. Scutari, "Distributed queueing games in interference-limited wireless networks," IEEE ICC, Budapest, Hungary, Jun. 2013.

[14] S. Elaydi, An Introduction to Difference Equations, 2nd Edition. Springer, New York, 1999. 